\title{
On the possibility of wireless battery charging in a gaseous-and-dusty mine
}

\author{
Irina Semykina ${ }^{1, *}$, Valery Zavyalov ${ }^{1,2}$, Evgeniy Dubkov ${ }^{1}$, and Amet-Han Veliliaev ${ }^{1}$ \\ ${ }^{1}$ Sevastopol State University, 299053, 33 Universitetskaya Street, Sevastopol, Russia \\ ${ }^{2}$ National Research Tomsk Polytechnic University, 634050, 30 Lenin Avenue, Tomsk, Russia
}

\begin{abstract}
The article outlines wireless battery charging as a promising direction for electric vehicles. There is the question raised of this technology applicability in the gaseous-and-dusty mine, and for such systems, the method for ignition risk evaluation is described. It is shown that the only potential ignition source is the charging area between the coils of the wireless charger. The authors formulate the objectives for studying the electromagnetic field in the charging area and propose variants of the mathematical description for this field. The research stages are listed which are necessary to substantiate the parameters and operating modes of wireless chargers that are safe for operating in the conditions of the gaseous-and-dusty mine.
\end{abstract}

\section{Introduction}

Underground mining is one of the priority areas of the industry [1]. A significant proportion of underground mines, which primarily are underground coalmines, are classified as gaseous-and-dusty mines that impose stringent requirements for ensuring explosion safety on the equipment used in its technological processes. At the same time, the direction of mining industry development assumes a continuous increase in the production level and labor productivity [2] that only increases the hazard level. To ensure safety in such conditions, it is required to develop and introduce modern innovative technologies and equipment for underground mining.

Considering the high power-to-weight ratio of mines, as well as the mobility of a significant part of the equipment [3], its important component providing energy storage are batteries. They are used not only in lighting devices and mine automation equipment but also in transport systems [4] such as mine electric locomotives, LHDs, etc. with an average battery capacity of tens of kilowatt-hours. At the same time, according to safety requirements, it is prohibited to disconnect batteries in the explosive zone, including charging. Disconnected batteries are charged on the surface in a specially equipped room. Thus, the technological operation of charging batteries for mine transport systems, due to safety requirements, is significantly complicated in comparison with surface electric transport with similar power and similar movement mode that increases operating costs of mine transport systems [5].

\footnotetext{
${ }^{*}$ Corresponding author: arinasemykina@gmail.com
} 
At the same time, according to Russian standards regulating explosion-proof electrical equipment, it is allowed to charge the batteries of mine electric locomotives from the contact wire using a power supply device. Those, battery charging in the explosive zone is not dangerous in principle [6] provided that there are no electrical commutations in the battery and load circuits. This circumstance allows us to consider the possibility of using charging systems for batteries of mine transport systems and other mobile equipment in gaseous-and-dusty mines which inherently do not require a wired connection to the grid, and therefore do not require disconnecting the batteries for charging that namely is wireless battery charging [7-9].

Wireless chargers for powerful batteries of electric vehicles have been developed abroad to a certain extent. In particular, there are practical recommendations for wireless chargers of electric vehicles [10], which determine the permissible criteria for technical and electromagnetic compatibility, EMF, minimum performance, safety, and testing for wireless chargers with a capacity from $3.6 \mathrm{~kW}$ to $150 \mathrm{~kW}$. At the same time, the applicability of these recommendations for gaseous-and-dusty mines, especially in terms of electromagnetic compatibility and safety, is questionable due to significant differences in operating conditions. Thus, for wireless chargers used in the explosive zone, it is advisable to conduct scientific research aimed at determining the boundary performance indicators ensuring that wireless chargers will not provoke the ignition and explosion of a hazardous explosive atmosphere.

\section{Problem statement}

Wireless power transfer used to wireless chargers is based on the physical principles of inductive coupling and magnetic resonance coupling. A typical structure of a wireless charger includes a high-frequency resonant converter with an inductive-capacitive circuit and inductively coupled transmitting and receiving coils as shown in Fig. 1 [11].

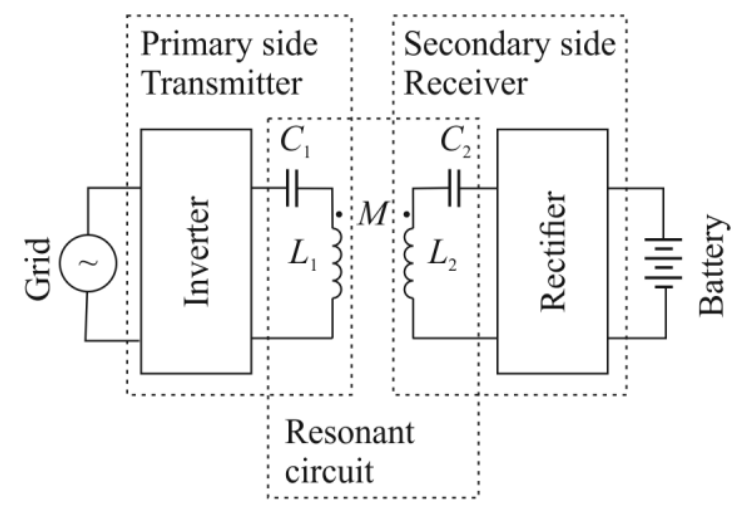

Fig. 1. Structural diagram of a wireless charger

The geometry and design of the transmitting and receiving coils affect the efficiency of wireless power transfer [12]. Depending on the configuration of the high-frequency resonant converter, the energy losses and the coupling coefficient will be different and the skin effect or other negative phenomena will manifest to a greater or lesser extent [13]. In normal operating mode, the wireless charger with optimized geometric and design parameters has more than $90 \%$ of efficiency [14]. However, the efficiency of wireless power transfer will drop off if the distance between the coils or their relative position change more than permissible, or if a foreign metal object appears between the coils. All these factors affect the dynamic state of the electromagnetic field in the charging area 
between the coils and can cause heating of conducting dust or metal particles in the hazardous explosive atmosphere, provoking ignition and explosion.

The most relevant way to prove the applicability of wireless chargers in the gaseousand-dusty mine and to establish the boundary performance indicators ensuring safety would be a physical experiment that provides creating a sample of a high-frequency resonant converter and its testing in a sealed chamber filled with a hazardous explosive atmosphere with a composition corresponding to the conditions of a particular mine. However, this approach is excessively expensive since the research will require several high-frequency resonant converters that differ in the topology of the inductive-capacitive circuit, as well as the geometry and design of the coils. In the case of ignition or explosion, the highfrequency resonant converter fails beyond recovery.

Considering the above, the ignition risk evaluation for high-frequency resonant converters of the wireless chargers in the gaseous-and-dusty mine can be made according to Russian standard [15].

\section{Methodology}

According to [15], the ignition risk is evaluated with the block diagram in Fig. 2 taking into account the appearance possibility of a hazardous explosive atmosphere inside and outside the equipment.

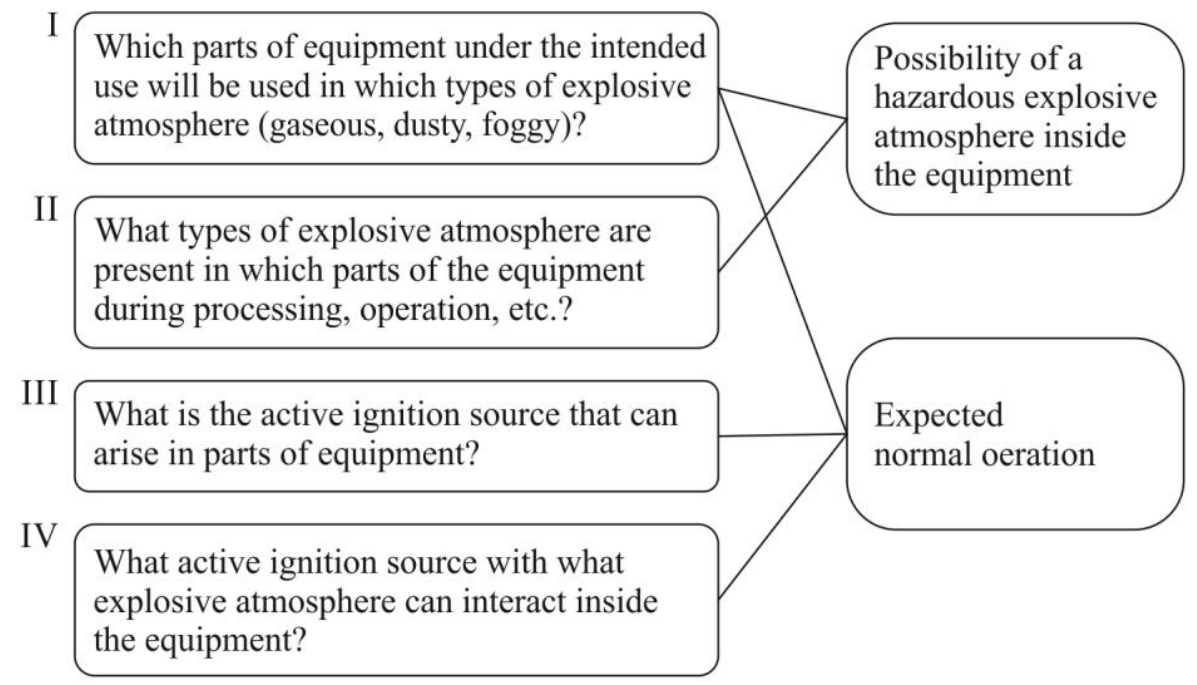

Fig. 2. Block diagram for ignition risk evaluation

To carry out such evaluation, let us consider an outline design diagram of a wireless charger system on the example of a mine electric locomotive, shown in Fig. 3, where the new structural elements are colored blue for ease of perception.

Since the system under consideration is supposed to be used in a hazardous explosive atmosphere of gaseous-and-dusty mine, all its elements must be equipped with explosion protection means. In this case, since a hazardous explosive atmosphere inside the elements of the system is possible, the secondary side and the rectifier, as well as the primary side and the inverter, must be placed in a flameproof enclosure "d". However, this type of explosion protection is not suitable for the transmitting and receiving coils, since according to [14], the presence of metal sheets between the coils reduces the efficiency of wireless power transfer to almost zero. Concurrently the same research shows that the presence of 
dielectric panels between the coils reduces the efficiency insignificantly, therefore, it is possible to use explosion protection by encapsulation " $\mathrm{m}$ " for the coils. Thus, the only potential ignition source is the charging area between the coils of the wireless charger, and the most dangerous operation mode is the ingress of foreign metal objects or conductive particles into it because they are concentrators of the electromagnetic field and their temperature will be the higher, the higher their magnetic permeability [16].

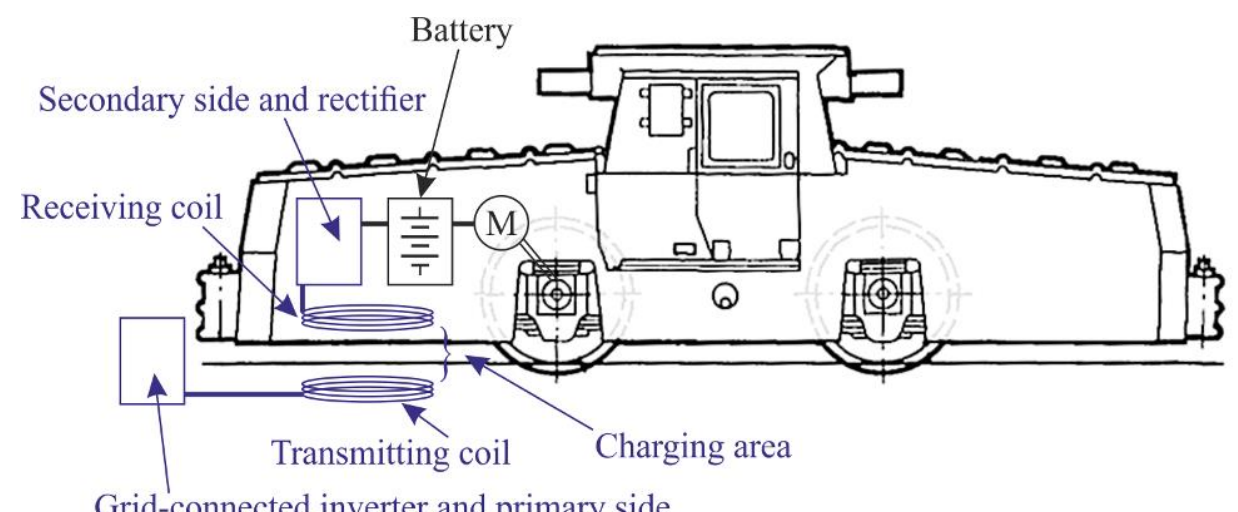

Fig. 3. Outline design diagram of a wireless charger system on the example of a mine electric locomotive

Based on the foregoing, the task of ignition risk evaluation for the wireless charger in the gaseous-and-dusty mine is to study the electromagnetic field interaction between the transmitting and receiving coils with foreign conductive objects located in the charging area. This task should be viewed from the following perspectives:

- Under what conditions will foreign objects have a significant impact on the efficiency of wireless power transfer?

- Will the elements of the electric locomotive's construction be heated by eddy currents, and on this basis, what should be the minimum distance from the receiving coil to the metal elements of the electric locomotive's construction?

- Are conductive objects in the charging area capable to heat up to the ignition temperatures of a hazardous explosive atmosphere?

To solve this problem, it is necessary to use a mathematical description of electromagnetic and thermal processes $[17,18]$.

\section{Mathematical description}

One of the approaches to solving the above problem is to expand the mathematical model of the resonant circuit with additional equivalent coils reflecting eddy currents in foreign conductive objects. The equivalent circuit is shown in Fig. 4

For a resonant circuit with a series topology of the capacitors in the primary and secondary sides, the extended mathematical model will be as follows:

$$
\begin{array}{lll}
\frac{d \psi_{1}}{d t}=u-u_{C 1}-i_{1} R_{1} ; & \frac{d \psi_{3}}{d t}=-i_{3} R_{3} ; & i_{1}=C_{1} \frac{d u_{C 1}}{d t} ; \\
\frac{d \psi_{2}}{d t}=-u_{C 2}-i_{2}\left(R_{2}+R_{L}\right) ; & \frac{d \psi_{N}}{d t}=-i_{N} R_{N} ; & i_{2}=C_{2} \frac{d u_{C 2}}{d t}
\end{array}
$$




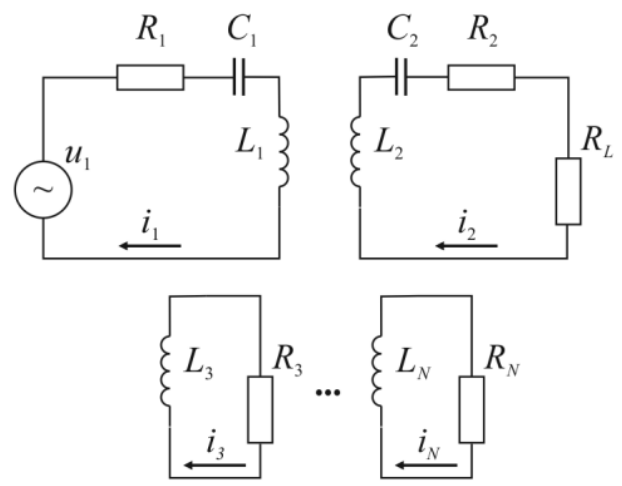

Fig. 4. The equivalent circuit of wireless charger with foreign conductive objects in the charging area where $u_{1}$ is the input voltage; $u_{C 1}$ is the capacitor voltage at the primary side; $u_{C 2}$ is the capacitor voltage at the secondary side; $i_{1}$ is the transmitting circuit current; $i_{2}$ is the receiving circuit current; $i_{3} \ldots i_{N}$ are eddy currents in foreign conductive objects; $\psi_{1} \ldots \psi_{N}$ are flux linkages respectively; $R_{1}, R_{2}, C_{1}, C_{2}$ - are primary side and secondary side resistance and capacitance consequently; $R_{L}$ - is the equivalent resistance of the battery; $L_{1}, L_{2}$ are transmitting and receiving coils inductance consequently; $R_{3} \ldots R_{N}, L_{3} \ldots L_{N}$ are equivalent resistances and inductances of foreign conductive objects which will substantially depend on their geometrical position relative to the transmitting and receiving coils and on the resonant frequency..

The flux linkage for the $i$-th coil of this system will be determined by the expressions:

$$
\psi_{i}=\sum_{j=1}^{N} i_{j} L_{i j}
$$

where the index $i$ denotes the coil in which the flux linkage is determined, and the index $j$ denotes the coil, which current contributes to the flux linkage of the $i$-th coil; $L_{i j}$ are the mutual inductances of the coils when $i \neq j$ and the self-inductance of the coil when $i=j$.

Based on the model (1), it is possible to assess the influence of foreign conductive objects on the efficiency of wireless power transfer, as well as to determine the heating power in the corresponding element:

$$
P_{N}=\int_{t} i_{N}^{2} R_{N} d t
$$

The obtained power can be used in further thermal calculations. It should be noted that this approach for calculating the thermal state is acceptable if the foreign conductive object has significantly smaller dimensions than the transmitting and receiving coils, and the electromagnetic field in the vicinity of the object can be considered uniform.

If this assumption is incorrect, from the standpoint of the ignition hazard it is advisable to consider another method for determining the power released in a conductive object:

$$
P_{\max }=\frac{d}{d t} \int_{v} W_{m} d v=\frac{d}{d t} \int_{v} \frac{B^{2}}{2 \mu_{0}} d v
$$

where $W_{m}$ is electromagnetic field energy, $B$ is electromagnetic induction, $\mu_{0}$ magnetic permeability of vacuum. 
The electromagnetic induction created by the currents of the coils in the resonant circuit can be determined using the Biot-Savart-Laplace Law:

$$
B_{r_{0}}=\frac{\mu_{0}}{4 \pi}\left[i_{1} \int_{l_{1}} \frac{d r_{1} \times\left(r_{0}-r_{1}\right)}{\left|r_{0}-r_{1}\right|^{3}}+i_{2} \int_{l_{2}} \frac{d r_{2} \times\left(r_{0}-r_{2}\right)}{\left|r_{0}-r_{2}\right|^{3}}\right]
$$

where $r_{0}$ is space coordinates of an element for which the electromagnetic induction is determined; $d r_{1}, d r_{2}$ are elements of the conductor of the transmitting and receiving coils with the currents $i_{1}$ and $i_{2}$ respectively; $r_{1}, r_{2}$ are space coordinates of an element of the transmitting and receiving coils consequently.

Thus, the maximum possible heating power in the foreign conductive object with volume $v$ located in the charging area can be calculated by the formula:

$$
P_{\max }=\int_{v} \frac{B^{2}}{2 \mu_{0}} d v=\frac{\mu_{0}}{32 \pi} \frac{d}{d t} \int\left[i_{v}(t) \int_{l_{1}} \frac{d r_{1} \times\left(r_{0}-r_{1}\right)}{\left|r_{0}-r_{1}\right|^{3}}+i_{2}(t) \int_{l_{1}} \frac{d r_{2} \times\left(r_{0}-r_{2}\right)}{\left|r_{0}-r_{2}\right|^{3}}\right]^{2} d v
$$

Considering that the joint analytical solution of (1), (2), and (6) can be very complicated, a possible solution to the described problem is the use of finite element models, which can be used to simulate electromagnetic and thermal fields in the charging area in a three-dimensional setting taking into account the physical properties of the materials of the transmitting and receiving coils and foreign conductive object, as well as the physical properties of a hazardous explosive atmosphere, in combination with the dynamic models of the electrical circuits of the wireless charger in the state space. Such models are considered, for example, in [19, 20].

\section{Conclusion}

The above approaches and models represent a preparatory stage of research aimed at assessing the possibility of using the wireless charger in the gaseous-and-dusty mine. In the future, using them, the following stages should be performed:

- Reasonable choice of specific type and model of mine battery equipment (mine electric locomotive, LHDs, etc.) with further development of circuitry and design technical solutions for the wireless charger, including the resonant circuit topology, as well as the geometry and design of the coils.

- Development of an integrated model of the wireless charger consisting of the supply network, the input power converter, the high-frequency inverter, the resonant circuit, the rectifier, the output filter, and the battery, in which a dynamic mathematical model in the state space is compiled for each element based on equivalent circuits, and for the transmitting and receiving coils the finite element model is created in a three-dimensional setting.

- Laboratory research of the electromagnetic and thermal fields of the wireless charger, during which the adequacy of the developed complex model is established.

- Conducting computational experiments on the developed integrated model for different operating modes of the wireless charger to identify the dependencies of electromagnetic compatibility and safety on the parameters of electromagnetic and thermal fields and external factors: the convergence and removal of the receiving and transmitting coils, their displacement with misalignment and location of foreign objects between the coils or in the immediate vicinity. 
- Justification of permissible parameters and operating modes of the wireless charger in the gaseous-and-dusty mine from the standpoint of safety, as well as the technical and electromagnetic compatibility.

The indicated research stages will make it possible to scientifically substantiate the applicability of wireless chargers in the gaseous-and-dusty mines which will increase the efficiency of mining by reducing operating costs in transport systems and will provide a transition to environmentally friendly technologies due to the prospects of replacing diesel mine transport to an electric one.

The article is supported by the internal grant of SevSU, project 27/06-31.

\section{References}

1. Underground Mining - Global Market Outlook (2019-2027), 173 (Stratistics Market Research Consulting Pvt Ltd, 2020)

2. J. Hirschi, Advances in Productive, Safe, and Responsible Coal Mining, 336 (Woodhead Publishing, 2018)

3. S.C. Walker, Mine Winding and Transport (Elsevier Science, 1988)

4. N. Jäderblom, From Diesel to Battery Power in Underground Mines, 75 (Luleå University of Technology, 2017)

5. M. A. H. Rafi, R. Rennie, J. Larsen, J. Bauman, IEEE Transportation Electrification Conference \& Expo (2020)

6. GMG Recommended Practices for Battery Electric Vehicles in Underground Mining (Global Mining Guidelines Group, 2018)

7. D.M. Vilathgamuwa, J.P.K. Sampath, Wireless Power Transfer for Electric Vehicles Present and Future Trends, 33 (Springer Science, 2015)

8. Ch. Qiu, K.T. Chau, Ch. Liu, C.C. Chan, World Electric Vehicle Symposium and Exhibition (2013)

9. Y. Gan, IOP Conference Series: Earth and Environmental Science (2020)

10. SAE J2954 Wireless Power Transfer for Light-Duty Plug-in. Electric Vehicles and Alignment Methodology (2019)

11. M. A. Houran, X. Yang, W. Chen, Electronics, 7, 296 (2018)

12. S. Li, C. C. Mi, IEEE Journal of Emerging and Selected Topics in Power Electronics, 3, 1, 4 (2015)

13. W. Shi, J. Deng, Z. Wang, X. Cheng, IEEE Access, 6, 14439 (2018)

14. I. Semykina, V. Zavyalov, V. Krylov, EDM 2020, 324 (2020)

15. GOST R 55816-2013 Methodology for the risk assessment of non-electrical equipment and components for intended use in potentially explosive atmospheres (2013) (in Russian)

16. S. Y. R. Hui, IEEE Electromagn. Compat. Mag., 7, 1, 78 (2018)

17. J. Pico, D. Hohlfeld, T. Bechtold, COMSOL Conference in Munich (2016)

18. D.V. Gretskih, A.I. Luchaninov, J.V. Vishniakova, V.A. Katrich, M.V. Nesterenko, International Seminar/Workshop on Direct and Inverse Problems of Electromagnetic and Acoustic Wave Theory (2018)

19. B. Olukotun, J. Partridge, R. Bucknall, Energies, 12, 3534 (2019)

20. L. Xiang, Z. Zhu, J. Tian, Y. Tian, IEEE Access 7, 44622 (2019) 